\title{
Distribution des points de petite hauteur dans les groupes multiplicatifs
}

\author{
FRANCESCO AMOROSO - SINNOU DAVID
}

\begin{abstract}
We prove a new lower bound for the height of points on a subvariety $V$ of a multiplicative torus, which lie outside the union of torsion subvarieties of $V$. Although lower bounds for the heights of these points where already known (decreasing multi-exponential function of the degree for Scmhidt and BombieriZannier, [Sch], [Bo-Za], and inverse monomial in the degree by the second author of this note and P. Philippon, [Da-Phi]), our method proves up to an $\varepsilon$ the sharpest conjectures that can be formulated.
\end{abstract}

Mathematics Subject Classification (2000): 11G10 (primary), 11J81, 14G40 (secondary).

\section{1. - Introduction}

Dans cet article, nous poursuivons l'étude des minorations de la hauteur normalisée des points d'une sous-variété d'un tore amorcée dans [Am-Da], [AmDa2] et [Am-Da3]. Soit $\iota: \mathbb{G}_{m}^{n} \hookrightarrow \mathbb{P}_{N}$ un plongement projectif induisant une compactification équivariante de $\mathbb{G}_{m}^{n}$ et $\hat{h}_{\iota}$ la hauteur normalisée associée. Parmi tous les minimums successifs algébriques pour la hauteur $\hat{h}_{\iota}(\cdot)$ sur $V$, on s'intéresse généralement plus particulièrement à trois invariants : le minimum essentiel, le minimum sur le complémentaire des translatés de sous-tores (de dimension au moins 1) et enfin le minimum «absolu», c'est à dire le minimum sur l'ouvert de Zariski $V^{\star}$ (en suivant les notations de [Da-Ph]) de $V$ :

$$
V^{\star}:=V \backslash \bigcup B,
$$

où la réunion est prise sur les variétés de torsion $B$ contenues dans $V$. Ici et dans la suite on appelera «variété de torsion» une réunion de translatés de sous-tores propres ${ }^{(1)}$ de $\mathbb{G}_{m}^{n}$ par des points de torsion. Dans le cas particulier

${ }^{(1)}$ Dans ce texte, «propre» signifie «strict» i.e. $\subsetneq$; on utilisera le mot «variété algébrique» pour indiquer un fermé de Zariski propre de $\mathbb{G}_{m}^{n}$, pas forcément irréductible ni équidimensionnel.

Pervenuto alla Redazione il 3 novembre 2003. 
où $V$ est un point d'ordre infini de $\mathbb{G}_{m}$, ces questions se réduisent au problème de Lehmer classique (confer [Le]).

Afin de simplifier les notations, les conjectures et les énoncés que l'on donnera par la suite, nous choisissons dès maintenant un plongement «privilégié» de $\mathbb{G}_{m}^{n}$ dans l'espace projectif, $\iota: \mathbb{G}_{m}^{n} \hookrightarrow \mathbb{P}_{n}$. La hauteur (normalisée) d'un point $\boldsymbol{\alpha}=\left(\alpha_{1}, \ldots, \alpha_{n}\right) \in \mathbb{G}_{m}^{n}$ est donc la hauteur de Weil logarithmique et absolue (avec la norme du sup aux places archimédiennes) $h(\boldsymbol{\alpha})$ du point projectif défini par $\left(1, \alpha_{1}, \ldots, \alpha_{n}\right)$. De même, par «degré» d'une sous-variété $W$ de $\mathbb{G}_{m}^{n}$, noté $\operatorname{deg}(W)$, on entendra le degré de l'adhérence de Zariski de $W$ dans $\mathbb{P}^{n}$.

Toujours pour alléger, nous identifierons dans la suite $\mathbb{G}_{m}^{n}$ (et ses sousvariétés) à $\mathbb{G}_{m}^{n}(\overline{\mathbb{Q}})$.

Comme nous l'avons fait observer dans [Am-Da], le degré n'est pas le bon invariant pour ce type de problèmes, mais l'indice d'obstruction, qui est plus fin, à savoir :

DÉFInition 1.1. Soit $V$ une sous-variété algébrique de $\mathbb{G}_{m}^{n}$ et soit $K$ une extension de $\mathbb{Q}$. On appelle indice d'obstruction de $V$ relatif à $K$, noté $\omega_{K}(V)$, le plus petit degré d'une hypersurface de $\mathbb{G}_{m}^{n}$ définie sur $K$ et passant par $V$.

On rappelle que pour toute sous-variété algébrique de $\mathbb{G}_{m}^{n}$ définie sur $K$ et $K$-irréductible (confer [Ch], Corollaire 2, Chapitre 1, page 8 et exemple 1 , page 9),

$$
\omega_{K}(V) \leq n \operatorname{deg}(V)^{1 / \operatorname{codim}(V)} .
$$

Dans [Am-Da] nous avons fait une conjecture (confer Conjecture 1.3 : on notera que dans op. cit. la quantité $\omega_{\mathbb{Q}}(\boldsymbol{\alpha})$ était notée $\left.\delta(\boldsymbol{\alpha})\right)$ qui généralise aux points de $\mathbb{G}_{m}^{n}$ celle de Lehmer et nous avons montré que cette dernière est vraie «à des facteurs log près». Ce résultat contient de plus une minoration du minimum essentiel d'une sous-variété algébrique de $\mathbb{G}_{m}^{n}$, définie sur $\mathbb{Q}$ et $\mathbb{Q}$-irréductible (confer [Am-Da3]). Rappelons ces résultats (confer [Am-Da] Théorème 1.5 et [Am-Da3], Corollaire 1.2) :

THÉORÈME 1.2. Pour tout entier $n \geq 1$ il existe deux nombres réels strictement positifs et effectivement calculables $c(n)$ et $\kappa(n)$, tels que pour tout élément $\boldsymbol{\alpha}$ de $\mathbb{G}_{m}^{n}$, dont les coordonnées sont multiplicativement indépendantes, on ait:

$$
h(\boldsymbol{\alpha}) \geq c(n)^{-1} \omega_{\mathbb{Q}}(\boldsymbol{\alpha})^{-1}\left(\log \left(3 \omega_{\mathbb{Q}}(\boldsymbol{\alpha})\right)\right)^{-\kappa(n)} .
$$

Soit de plus $V$ une sous-variété algébrique de $\mathbb{G}_{m}^{n}$, définie sur $\mathbb{Q}$ et $\mathbb{Q}$-irréductible qui n'est pas contenue dans une sous-variété de torsion de $\mathbb{G}_{m}^{n}$. Alors l'ensemble des points $\boldsymbol{\alpha} \in V$ tels que

$$
h(\boldsymbol{\alpha})<c(n)^{-1} \omega_{\mathbb{Q}}(V)^{-1}\left(\log \left(3 \omega_{\mathbb{Q}}(V)\right)\right)^{-\kappa(n)}
$$

n'est pas Zariski dense dans $V$. 
On peut en fait prendre dans ce théorème $\kappa(n):=2 n(n+1) !^{n}-1$. On notera que la valeur numérique indiquée dans [Am-Da] est erronée ; au cours de la preuve du Théorème 1.5 ci-dessous, nous indiquerons les modifications à apporter pour corriger cette faute da calcul.

Dans cet article on se propose de donner des résultats plus précis par rapport au Théorème 1.2. Soit $V$ une sous-variété algébrique de $\mathbb{G}_{m}^{n}$, définie sur $\mathbb{Q}$ et $\mathbb{Q}$-irréductible. Remarquons d'abord que l'on peut rien dire sur la hauteur des points d'une sous-variété de torsion $B$ de $V$ : en effet, on peut trouver dans $B$ des points de torsion et même des points de hauteur positive mais arbitrairement petite (par rapport à $V$ ). Par contre, sur l'ouvert $V^{*}$, la hauteur est minorée uniquement en fonction de $V$ et, dans [Da-Ph], le second auteur et P. Philippon ont montré que la hauteur des points de $V^{*}$ est minorée par:

$$
\left(2^{2 n+4 d+25} \operatorname{deg}(V)(\log (\operatorname{deg}(V)+1))^{3}\right)^{-7^{d}},
$$

où $d$ est la dimension de $V$. Cette minoration, même si elle améliore de façon spectaculaire les bornes précédemment connues (E. Bombieri-U. Zannier, [BoZa] et W. Schmidt, [Sch]), semble loin d'être optimale.

Les résultats obtenus d'une part dans [Am-Da] et [Am-Da3] et d'autre part la recherche de la bonne dépendance en le degré du plongement de $\mathbb{G}_{m}^{n}$ dans un projectif nous ont amenés à formuler une conjecture pour le comportement du minimum de la hauteur dans $V^{\star}$ dans [Am-Da4] (voir la Conjecture 1.12). Cette conjecture est renforcée par les résultats obtenus en dimension 1 par le premier auteur et R. Dvornicich (confer [Am-Dv]) et par ailleurs avec U. Zannier (confer $[\mathrm{Am}-\mathrm{Za}])$. Une version simplifiée de cette conjecture peut s'énoncer comme suit :

Conjecture 1.3. Soit $V$ une sous-variété algébrique de $\mathbb{G}_{m}^{n}$ définie sur $\mathbb{Q}^{\mathrm{ab}}$. Supposons que $V$ soit une intersection d'hypersurfaces de $\mathbb{G}_{m}^{m}$ définies sur $\mathbb{Q}^{\text {ab }}$ et de degré au plus $\delta$. Alors, pour tout point $\boldsymbol{\alpha}$ de $V^{*}$ on a :

$$
h(\boldsymbol{\alpha}) \geq c(n)^{-1} \delta^{-1},
$$

où $c(n)$ est un nombre réel $>0$.

On remarquera que toute sous-variété algébrique $\mathbb{G}_{m}^{n}$, définie sur $\mathbb{Q}^{\mathrm{ab}}$ et $\mathbb{Q}^{\mathrm{ab}}$ irréductible est intersection d'hypersurfaces de $\mathbb{G}_{m}^{n}$ de degré au plus $\operatorname{deg}(V)$; cette conjecture implique donc en particulier la minoration suivante pour les points $\alpha \in V^{*}$ :

$$
\forall \boldsymbol{\alpha} \in V^{\star}(\overline{\mathbb{Q}}), \quad h(\boldsymbol{\alpha}) \geq c(n)^{-1} \operatorname{deg}(V)^{-1} .
$$

Par ailleurs on aurait pu espérer que la minoration optimale dans la conjecture précédente fasse intervenir l'indice d'obstruction $\omega_{\mathbb{Q}^{\mathrm{ab}}}(V)$ en lieu et place de la régularité $\delta$, mais ceci est faux, comme l'exemple suivant le montre. 
Soit $\alpha$ un nombre algébrique de hauteur strictement positive mais suffisamment petite tel que $\mathbb{Q}(\alpha)$ et $\mathbb{Q}^{\mathrm{ab}}$ soient linéairement disjoints et soit $P(t) \in \mathbb{Q}[t]$ son polynôme minimal. Notons

$$
V=\left\{\left(x_{1}, x_{2}, x_{3}\right) \in \mathbb{G}_{m}^{3} \quad \text { tel que } x_{1}^{2}+x_{1}^{3}-x_{2}-x_{3}=0=P\left(x_{1}\right)\right\} .
$$

On vérifie que $\alpha=\left(\alpha, \alpha^{2}, \alpha^{3}\right) \in V$ et que $\alpha$ n'appartient à aucune sousvariété de torsion contenue dans $V$. Par ailleurs $\omega_{\mathbb{Q}}$ ab $(V)=3$; l'inégalité $h(\boldsymbol{\alpha}) \geq c(n)^{-1} \omega_{\mathbb{Q}^{\mathrm{ab}}}(V)^{-1}$ est donc fausse.

Dans la direction de la Conjecture 1.3, nous allons établir le résultat suivant :

THÉORÈME 1.4. Pour tout entier $n \geq 1$ il existe un nombre réel strictement positif c (n), effectivement calculable, tel que la propriété suivante soit vraie. Soit $V$ une sous-variété algébrique de $\mathbb{G}_{m}^{n}$, définie sur une extension cyclotomique $K$ de $\mathbb{Q}$ $e t^{(2)}$ supposons que $V$ soit une intersection d'hypersurfaces de $\mathbb{G}_{m}^{n}$ définies sur $K$ et de degré au plus $\delta$. Alors, pour tout point $\boldsymbol{\alpha} \in V^{\star}$, on a :

$$
h(\boldsymbol{\alpha}) \geq c(n)^{-1} \delta^{-1}(\log (3[K: \mathbb{Q}] \delta))^{-\kappa(n)},
$$

où $\kappa(n):=2 n(n+1) !^{n}-1$. En particulier, si $V$ est définie par des équations à coefficients rationnels de degré $\leq \delta$, on a :

$$
h(\boldsymbol{\alpha}) \geq c(n)^{-1} \delta^{-1}(\log (3 \delta))^{-\kappa(n)} .
$$

pour tout point $\alpha \in V^{\star}$.

REMARQues.

(i) la dépendance en $\omega_{K}(\boldsymbol{\alpha})$ dans la minoration de la hauteur du Théorème 1.4 est très satisfaisante (à l'exception peut-être des valeurs de $c^{\prime}(n)$ et $\kappa(n)$ ) ; en particulier ce théorème est optimal à un $\epsilon$-près si $K=\mathbb{Q}$. Par ailleurs, on devrait pouvoir faire disparaitre la dépendance en le degré $[K: \mathbb{Q}]$, dans le facteur logarithmique, dans l'esprit de [Am-Za]. Pour ce faire, il conviendrait sans doute de parvenir à combiner les techniques de [Am-Za] et $[\mathrm{Am}-\mathrm{Da}]$.

(ii) le Théorème 1.4 est un raffinement du Théorème 1.5 de [Am-Da]. En effet, si $\boldsymbol{\alpha} \in \mathbb{G}_{m}^{n} \backslash\left(\mathbb{G}_{m}^{n}\right)_{\text {tors }}$, notons $\omega_{\mathbb{Q}}^{*}(\boldsymbol{\alpha})$ le plus petit degré d'une hypersurface $Z$ de $\mathbb{G}_{m}^{n}$ définie sur $\mathbb{Q}$ et telle que $\alpha \in Z^{*}$. Par définition, on a $\omega_{\mathbb{Q}}^{*}(\boldsymbol{\alpha}) \geq \omega_{\mathbb{Q}}(\boldsymbol{\alpha})$ avec égalité si les coordonnées du point $\boldsymbol{\alpha}$ sont multiplicativement indépendantes. Le théorème qui précède donne alors en particulier la minoration

$$
h(\boldsymbol{\alpha}) \geq c(n)^{-1} \omega_{\mathbb{Q}}^{*}(\boldsymbol{\alpha})^{-1}\left(\log \left(3 \omega_{\mathbb{Q}}^{*}(\boldsymbol{\alpha})\right)^{-\kappa(n)} ;\right.
$$

${ }^{(2)}$ On remarquera que la variété $V$ n'est pas supposé $K$-irréductible. 
(iii) dans le cas particulier où $n=1$, la variété $V$ est de la forme : $V=$ $\cup_{\sigma \in \operatorname{Gal}(\bar{K} / K)} \sigma(\alpha)$ où $\alpha \in \mathbb{G}_{m}$ et $V^{\star}$ est non vide si et seulement si $\alpha$ est d'ordre infini. Dans ce cas, le Théorème 1.4 montre que

$$
h(\alpha) \geq \frac{c(1)}{[K(\alpha): K]}(\log (3[K(\alpha): \mathbb{Q}]))^{-3} .
$$

Le Théorème 1.4 est donc une version faible du résultat principal (sauf bien sur si $[K: \mathbb{Q}]$ est assez petit devant $[K(\alpha): K])$ de $[\mathrm{Am}-\mathrm{Za}]$ qui assure lui :

$$
h(\alpha) \geq \frac{c}{[K(\alpha): K]}\left(\frac{\log (3[K(\alpha): K])}{\log \log (3[K(\alpha): K])}\right)^{-13}
$$

Le Théorème 1.2 se présente comme une dichotomie : ou bien la hauteur de $\boldsymbol{\alpha}$ est assez grande, ou bien les cordonnées de $\boldsymbol{\alpha}$ sont multiplicativement liées, i.e. $\boldsymbol{\alpha}$ est dans une sous-variété de torsion. Comme souvent en géométrie diophantienne, cette dichotomie peut être quantifiée, et l'on peut dans le cas dégénéré majorer le degré d'une variété de torsion passant par $\boldsymbol{\alpha}$. Cette information est bien souvent porteuse d'applications arithmétiques de nature distinctes de celles que l'on peut tirer du reste de l'énoncé, et en fait nous déduirons même le Théorème 1.4 d'un énoncé quantitatif de ce type :

THÉORÈME 1.5. Pour tout entier $n \geq 1$ il existe un nombre réel strictement positif $c^{\prime}(n)$, effectivement calculable, tel que la propriété suivante soit vraie. Soit $K$ une extension cyclotomique de $\mathbb{Q}$ et $\boldsymbol{\alpha}$ un élément de $\mathbb{G}_{m}^{n}$. Notons :

$$
\kappa(n):=2 n(n+1) !^{n}-1 \quad \text { et } \quad \mu(n):=2 n\left((n+1) !^{n}+(n+1) !^{n-1}-2 .\right.
$$

$S i$

$$
h(\boldsymbol{\alpha})<c^{\prime}(n)^{-1} \omega_{K}(\boldsymbol{\alpha})^{-1}\left(\log \left(3[K: \mathbb{Q}] \omega_{K}(\boldsymbol{\alpha})\right)\right)^{-\kappa(n)},
$$

alors, il existe une sous-variété de torsion $B$ contenant $\alpha$, définie sur $K$ et $K$ irréductible, telle que :

$$
(\operatorname{deg} B)^{1 / \operatorname{codim}(B)} \leq c^{\prime}(n) \omega_{K}(\boldsymbol{\alpha})\left(\log \left(3[K: \mathbb{Q}] \omega_{K}(\boldsymbol{\alpha})\right)\right)^{\mu(n)} .
$$

Le déroulement de la preuve du Théorème 1.5 est très similaire à celui du théorème principal de [Am-Da]. Nous indiquerons dans la suite les modifications nécessaires pour montrer le Théorème 1.5 ; nous ferons référence aussi suivant que possible aux résultats et aux démonstrations de [Am-Da].

Au Paragraphe 2 nous avons développé la machinerie de transcendance : lemme de Siegel, estimation du rang, extrapolation, choix des paramètres et lemme de zéros. À la différence de [Am-Da], nous aurons besoin d'une version du lemme de Siegel sur un corps de nombre (en l'occurrence, une extension cyclotomique de $\mathbb{Q}$ ), d'où la présence d'un discriminant. L'extrapolation repose sur la généralisation du lemme clef de Dobrowolski (confer [Do]) déjà démontrée 
dans [Am-Da] ; cependant nous aurons besoin, pour faire apparaitre la quantité $\omega_{K}(\boldsymbol{\alpha})^{-1}$ dans le terme principal de notre inégalité, de composer isogénies (i.e. élévation à la puissance $p$-ième) et conjugaisons (i.e. par des éléments de Frobenius du groupe de Galois). Le lemme de zéros est, à quelques détail près, le même que celui de [Am-Da]. Comme dans op. cit. on montrera à la fin du Paragraphe 2 que «si la conclusion du Théorème 1.5 est fausse, alors on peut trouver des petits multiples de $\boldsymbol{\alpha}$ pour lesquels l'indice d'obstruction $\omega_{K}\left(\boldsymbol{\alpha}^{l}\right)$ est pathologiquement petit par rapport à $\boldsymbol{\alpha} »$. Malheureusement, cette proposition ne suffit pas plus que dans loc. cit. pour conclure la preuve : comme dans [AmDa] nous aurons besoin d'un argument de descente, que l'on trouvera dans le Paragraphe 3. Enfin, les Théorèmes 1.5 et Théorème 1.4 seront démontrés au Paragraphe 4.

C. Pointreau nous a fait part de ses remarques sur une première version de ce texte et nous a permis d'améliorer la présentation de nombreux points. C'est un plaisir pour nous de pouvoir le remercier chaleureusement ici.

\section{1. - Notations et rappels}

Soit $n$ un entier $\geq 1$; dans toute la suite du texte, nous plongerons $\mathbb{G}_{m}^{n}$ naturellement dans $\mathbb{P}^{n}:$ par «degré» d'une sous-variété $W$ de $\mathbb{G}_{m}^{n}$, noté $\operatorname{deg}(W)$, on entendra le degré de l'adhérence de Zariski de $W$ dans $\mathbb{P}^{n}$. Dans la suite du texte, on fixe également une extension cyclotomique $K$ de $\mathbb{Q}$, de degré $D$ et de conducteur $\mathfrak{f}_{K}$; en particulier, $K=\mathbb{Q}(\zeta)$ où $\zeta$ est une racine $\mathfrak{f}_{K}$-ième primitive de 1 , et $\varphi\left(\mathfrak{f}_{K}\right)=D$, où $\varphi(\cdot)$ est l'indicatrice d'Euler. Soit $p$ un premier ne divisant pas $\mathfrak{f}_{K}$; ce premier n'est donc pas ramifié dans $K$. On notera $\phi_{p} \in \operatorname{Gal}(K / \mathbb{Q})$ l'automorphisme de Frobenius relatif à $p$. Par abus de notation on posera : $\phi_{1}=$ Id. Enfin, si $P$ est un polynôme à coefficients dans $\overline{\mathbb{Q}}$ et si $\sigma \in \operatorname{Gal}(\overline{\mathbb{Q}} / \mathbb{Q})$, on notera $P^{\sigma}$ le polynôme dont les coefficients sont les conjugués par $\sigma$ de ceux de $P$.

Comme dans [Am-Da], la preuve se fera par «extrapolation» sur des multiplication par des premiers $p$; nous aurons besoin d'éviter des entiers «exceptionnels» attachés à une sous-variété de $\mathbb{G}_{m}^{n}$, pour éviter des comportements pathologiques dans les diverses estimations, ce qui motive la définition suivante (comparer avec la définition 2.2 de loc. cit.) :

Définition 1.6. Soit $V$ une sous-variété algébrique propre de $\mathbb{G}_{m}^{n}$, définie sur $K$ et $K$-irréductible, notons $W$ une de ses composantes géométriquement irréductible. On note $E(V)$ l'ensemble des entiers relatifs $l$ qui satisfont au moins une des propriétés suivantes :

(i) $l$ n'est pas premier avec $\mathfrak{f}_{K}$;

(ii) il existe $\sigma \in \operatorname{Gal}(\overline{\mathbb{Q}} / \mathbb{Q})$ tel que $\sigma(W) \neq W$ et $\sigma([l] W)=[l] W$;

(iii) $\operatorname{deg}([l] W)<\operatorname{deg}(W)$.

Les propriétés principales de l'ensemble $E(V)$ sont résumées dans la proposition suivante (confer [Am-Da], Proposition 2.4) : 
Proposition 1.7. Soit $V$ une sous-variété propre de $\mathbb{G}_{m}^{n}$, définie sur $K$ et $K$-irréductible, de dimension $d$. On a alors :

(i) $\mid E(V) \cap\{p$ premier $\} \mid \leq \frac{d+2}{\log 2} \log ([K: \mathbb{Q}] \operatorname{deg}(V))$.

(ii) Soient l et l' deux entiers n'appartenant pas à $E(V)$. Alors : $l l^{\prime} \notin E(V)$.

DÉmonstration. Le nombre de premiers $p$ divisant $\mathfrak{f}_{K}$ est majoré par :

$$
\frac{2 \log \varphi\left(\mathfrak{f}_{K}\right)}{\log 2}=\frac{2 \log [K: \mathbb{Q}]}{\log 2} .
$$

Par ailleurs, la preuve de la Proposition 2.4, point (i), de [Am-Da] montre que l'ensemble des premiers $p$ pour lesquels $\operatorname{deg}([p] W)<\operatorname{deg}(W)$ est de cardinal au plus $(d+1)(\log 2)^{-1} \log \operatorname{deg}(W)$. Enfin, le Lemme 2.3, point (ii), de op. cit. montre que le nombre de premiers $p$ tels qu'il existe $\sigma \in \mathrm{Gal}(\overline{\mathbb{Q}} / \mathbb{Q})$ avec $\sigma(W) \neq W$ et $\sigma([p] W)=[p] W$ est borné par $(\log 2)^{-1} \log k$, où $k$ est le nombre des composantes $\overline{\mathbb{Q}}$-irréductibles de $V$. On a donc :

$$
\begin{aligned}
E(V) \cap\{p \text { premier }\} & \leq \frac{2}{\log 2} \log [K: \mathbb{Q}]+\frac{d+1}{\log 2} \log \operatorname{deg}(W)+\frac{1}{\log 2} \log k \\
& \leq \frac{d+2}{\log 2} \log ([K: \mathbb{Q}] k \operatorname{deg}(W)) \\
& =\frac{d+2}{\log 2} \log ([K: \mathbb{Q}] \operatorname{deg}(V)),
\end{aligned}
$$

ce qui montre le point (i). La preuve du point (ii) est très similaire à celle du point correspondant de la Proposition 2.4 de op. cit. et la proposition suit.

\section{2. - La transcendance}

La stratégie de la preuve du Théorème 1.5 est la suivante. On se donne un point $\alpha$ de $\mathbb{G}_{m}^{n}$ satisfaisant les hypothèses du Théorème 1.5. On construit alors un polynôme de petit degré et de hauteur contrôlée s'annulant en $\alpha$ avec une forte multiplicité (c'est l'objet du Paragraphe 2), puis on en déduit que ce dernier s'annule en de très nombreux multiples de $\alpha$ (au Paragraphe 2.2). Un lemme de zéros suivi d'un argument de «descente» permet ensuite de conclure.

\section{1. - La construction de la fonction auxiliaire}

Contrairement à [Am-Da], où la la fonction auxiliaire est rationnelle sur $\mathbb{Q}$, il convient ici de construire cette dernière sur l'extension cyclotomique $K$ (sinon, on ne parviendrait pas à remplacer $\omega_{\mathbb{Q}}(\boldsymbol{\alpha})$ par $\omega_{K}(\boldsymbol{\alpha})$ dans le résultat). Cette première modification par rapport à $o p$. cit. est facile, mais fait intervenir le discriminant de $K / \mathbb{Q}$ (lemme de Bombieri-Vaaler). 
Proposition 2.1. Soient $L$ et $T$ deux entiers $\geq 1$ vérifiant les inégalités :

$$
2 \omega_{K}(\boldsymbol{\alpha}) T \leq L, \quad \text { et } \quad h(\boldsymbol{\alpha}) \leq \frac{T \log (L+1)}{L} .
$$

Il existe alors un polynôme non nul $F \in K\left[x_{1}, \ldots, x_{n}\right]$ de degré $\leq L$, s'annulant en $\alpha$ à un ordre $\geq T$ tel que :

$$
h(F) \leq \log [K: \mathbb{Q}]+\frac{4^{n+1} \omega_{K}(\boldsymbol{\alpha}) T^{2} \log (L+1)}{L} .
$$

Démonstration. La preuve de cette proposition est identique à celle de la proposition correspondante de [Am-Da] (Proposition 4.2) : il suffit de remplacer partout le corps des nombres rationnels par $K$. Dans l'application du Théorème 8 de [Bo-Va], il faut bien sûr prendre en compte le discriminant de ce corps ; cela conduit à la majoration suivante pour $h(F)$ :

$$
h(F) \leq \log c(K)+\frac{4^{n+1} \omega_{K}(\boldsymbol{\alpha}) T^{2} \log (L+1)}{L},
$$

où

$$
c(K)=\left\{\left(\frac{2}{\pi}\right)^{r_{2}} \sqrt{|\operatorname{disc}(\mathrm{K})|}\right\}^{1 /[K: \mathbb{Q}]},
$$

(et où $r_{1}$ est le nombre de places réelles de $K$ et $r_{2}$ le nombre de places complexes, et bien sûr $\left.[K: \mathbb{Q}]=r_{1}+2 r_{2}\right)$. Comme $K$ est cyclotomique, si $K \neq \mathbb{Q}$, on a, $r_{2}=[K: \mathbb{Q}] / 2$ et $|\operatorname{disc}(K)|^{1 /[K: \mathbb{Q}]} \leq \mathfrak{f}_{K}$. On en tire $c(K) \leq$ $\sqrt{2 \mathfrak{f}_{K} / \pi} \leq \varphi\left(\mathfrak{f}_{K}\right)=[K: \mathbb{Q}]\left(\operatorname{car} \varphi(n) \geq \sqrt{n}\right.$ si $n \geq 3, n \neq 6$ et $\mathfrak{f}_{K}$ ne peut être égal à 6 ou $\leq 2$ si $K \neq \mathbb{Q}$ ). Si $K=\mathbb{Q}$, on a bien en tendu $c(K)=1=[K: \mathbb{Q}]$.

\section{2. - Extrapolation}

L'extrapolation suit dans les grandes lignes celle de [Am-Da] (et Dobrowolski). On montre que la fonction construite au paragraphe précédent s'annule également en de nombreux multiples de $\alpha$. Une différence tout de même à signaler : comme on n'est plus sur le corps des rationnels, il convient tout d'abord de généraliser convenablement le Théorème 3.1 de op. cit. L'argument se transporte bien car d'une part l'extension $K / \mathbb{Q}$ est abélienne et d'autre part car on se restreint à des premiers non ramifiés dans $K$ (voir la définition 1.6).

THÉORÈME 2.2. Soit $K$ une extension cyclotomique de $\mathbb{Q}, F \in \overline{\mathbb{Q}}\left[x_{1}, \ldots, x_{n}\right]$ de degré $\leq L$ et nul à un ordre $\geq T$ sur $\sigma(\boldsymbol{\alpha})$ pour tout $\sigma \in \mathrm{Gal}(\overline{\mathbb{Q}} / K)$. Pour tout nombre premier $p \in \mathbb{Z}$ ne divisant pas $\mathfrak{f}_{K}$ et pour toute valuation ultramétrique $v$ de $\overline{\mathbb{Q}}$ divisant $p$, on a la majoration :

$$
\left|F^{\phi_{p}}\left(\boldsymbol{\alpha}^{p}\right)\right|_{v} \leq p^{-T}|F|_{v} \max \left\{1,\left|\alpha_{1}\right|_{v}, \ldots,\left|\alpha_{n}\right|_{v}\right\}^{p L} .
$$


Démonstration. On peut supposer que $|F|_{v}=1$. On suit la preuve du Théorème 3.1 de [Am-Da] en remplaçant l'anneau $\mathbb{Z}$ par l'anneau $\mathcal{O}_{K}$ des entiers de $K$. Comme dans loc. cit., on peut supposer que $\alpha_{1}, \ldots, \alpha_{n}$ sont des entiers algébriques engendrant l'anneau des entiers de $K\left(\alpha_{1}, \ldots, \alpha_{n}\right)$ en tant que $\mathcal{O}_{K}$-module. Cette preuve montre alors que l'on peut écrire :

$$
F=\sum_{\mathbf{i} \in \mathbb{N} T} f_{i_{1}} \ldots f_{i_{T}}
$$

où les $f_{i_{l}}$ sont des polynômes de $\mathcal{O}_{K}$ nuls en $\boldsymbol{\alpha}$.

Comme l'extension $K / \mathbb{Q}$ est abélienne, pour tout élément $a \in \mathcal{O}_{K}$ et tout premier $\mathfrak{p}$ au dessus de $p, \phi_{p}(a)-a^{p} \in \mathfrak{p}$, par suite,

$$
\phi_{p}(a)-a^{p} \in \bigcap_{\mathfrak{p} \mid p} \mathfrak{p}=p \mathcal{O}_{K}
$$

Le petit théorème de Fermat s'applique donc aux $f_{i_{l}}$ mutatis mutandis comme dans $\mathbb{Z}$ et donne alors :

$$
f_{i_{l}}^{\phi_{p}}\left(x_{1}^{p}, \ldots, x_{n}^{p}\right)=f_{i_{l}}(\mathbf{x})^{p}+p g_{i_{l}}(\mathbf{x}), \quad g_{i_{l}}(\mathbf{x}) \in \mathcal{O}_{K}[\mathbf{x}] .
$$

On en tire :

$$
F^{\phi_{p}}\left(\boldsymbol{\alpha}^{p}\right)=p^{T} \sum_{\mathbf{i} \in \mathbb{N}^{T}} g_{i_{1}}(\boldsymbol{\alpha}) \ldots g_{i_{T}}(\boldsymbol{\alpha})=p^{T} \beta
$$

pour un certain entier algébrique $\beta$. Donc : $\left|F^{\phi_{p}}\left(\boldsymbol{\alpha}^{p}\right)\right|_{v} \leq p^{-T}$, ce qui établit le Théorème 2.2.

On en déduit comme dans [Am-Da] l'analogue suivant du Lemme 4.3 de op. cit. :

LemMe 2.3. Soient $L$ et $T$ deux entiers $\geq 1$ et $p$ un nombre premier ne divisant pas $\mathfrak{f}_{K}$. On note

$$
T^{*}:=2^{-1}\left(1+\frac{(n+1) \log (L+1)}{\log p}\right)^{-1} \times T
$$

et on suppose $T^{*} \geq 1$. Soit de plus $F \in K\left[x_{1}, \ldots, x_{n}\right]$ un polynôme de degré $\leq L$, nul en un point $\alpha \in \mathbb{G}_{m}^{n}$ à un ordre $\geq T$; supposons aussi :

$$
h(F) \leq \frac{1}{4} T \log p \quad \text { et } \quad h(\alpha)<\frac{T \log p}{4 p L} .
$$

Alors le polynôme $F^{\phi_{p}}$ est nul en $\boldsymbol{\alpha}^{p}$ à un ordre $\geq T^{*}$. 
En utilisant $r$ fois le Lemme 2.3, on en tire :

Lemme 2.4. Soient $L, T$ et $r$ trois entiers $\geq 1$ et $p_{1}, \ldots, p_{r}$ des nombres premiers ne divisant pas $\mathfrak{f}_{K}$. Pour $s=0, \ldots, r$, on note

$$
T_{s}:=2^{-s} \prod_{j=1}^{s}\left(1+\frac{(n+1) \log (L+1)}{\log p_{j}}\right)^{-1} \times T
$$

(avec la convention $T_{0}=T$ ) et on suppose $T_{r} \geq 1$. Soit de plus $F \in K\left[x_{1}, \ldots, x_{n}\right]$ un polynôme de degré $\leq L$, nul à un ordre $\geq T$ en un point $\boldsymbol{\alpha}$ de $\mathbb{G}_{m}^{n}$. Supposons :

$$
h(F) \leq \frac{1}{4} T_{r-1} \min _{1 \leq j \leq r} \log p_{j}
$$

et

$$
h(\boldsymbol{\alpha})<\frac{T_{r-1} \min _{1 \leq j \leq r} \log p_{j}}{4 L p_{1} \ldots p_{r}} .
$$

Alors le polynôme $F^{\phi_{p_{1}} \ldots \phi_{p_{r}}}$ est nul en $\boldsymbol{\alpha}^{p_{1} \ldots p_{r}} \grave{a}$ un ordre $\geq T_{r}$.

DÉmonstration. Montrons cet énoncé par récurrence sur $r$ (le cas $r=1$ étant le Lemme 2.3). Soit donc $r$ un entier $\geq 2$; soient aussi $L$ et $T$ deux entiers $\geq 1$ et $p_{1}, \ldots, p_{r}$ des nombres premiers ne divisant pas $\mathfrak{f}_{K}$ et soit enfin $F \in K\left[x_{1}, \ldots, x_{n}\right]$ un polynôme de degré $\leq L$, nul à un ordre $\geq T$ en un point $\boldsymbol{\alpha}$ de $\mathbb{G}_{m}^{n}$ qui satisfait de plus les conditions (1) et (2). En particulier on a :

$$
h(F) \leq \frac{1}{4} T_{r-2} \min _{1 \leq j \leq r-1} \log p_{j} \quad \text { et } \quad h(\alpha)<\frac{T_{r-2} \min _{1 \leq j \leq r-1} \log p_{j}}{4 L p_{1} \ldots p_{r-1}} ;
$$

par récurrence le polynôme $F^{\phi_{p_{1}} \ldots \phi_{p_{r-1}}}$ est donc nul en $\boldsymbol{\alpha}^{p_{1} \ldots p_{r-1}}$ à un ordre $\geq T_{r-1}$. On a $h\left(F^{\phi_{p_{1}} \ldots \phi_{p_{r-1}}}\right)=h(F)$; par ailleurs, l'hypothèse (2) et la relation $h\left(\boldsymbol{\alpha}^{p_{1} \ldots p_{r}}\right)=p_{1} \ldots p_{r} h(\boldsymbol{\alpha})$ impliquent :

$$
h\left(\boldsymbol{\alpha}^{p_{1} \ldots p_{r}}\right)<\frac{T_{r-1} \min _{1 \leq j \leq r} \log p_{j}}{4 L} .
$$

On déduit alors du Lemme 2.3 (où l'on a remplacé $T^{*}, p, \alpha$ et $F$ par $T_{r-1}$, $p_{r}, \boldsymbol{\alpha}^{p_{1} \ldots p_{r-1}}$ et $F^{\phi_{p_{1} \ldots \phi_{p_{r-1}}}}$ respectivement) que le polynôme $F^{\phi_{p_{1}} \ldots \phi_{p_{r}}}$ est nul en $\boldsymbol{\alpha}^{p_{1} \ldots p r}$ à un ordre $\geq T^{*}$, où :

$$
T^{*}=2^{-1}\left(1+\frac{(n+1) \log (L+1)}{\log p_{r}}\right)^{-1} \times T_{r-1}=T_{r} .
$$

Le Lemme 2.4 est donc entièrement établi.

Le Lemme 2.4 est l'analogue du Lemme 4.4 de [Am-Da] ; c'est au niveau de la déduction du Lemme 4.4 de op. cit. à partir du Lemme 4.3 qu'une erreur s'est glissée. Les hypothèses (11) et (12) du Lemme 4.4 doivent être remplacées par les inégalités (1) et (2) ci-dessus. Cette erreur affecte le choix des paramètres (Paragraphe 5 de loc. cit.) et donc la valeur numérique de la constante $\kappa(n)$ dans le théorème principal (Théorème 1.5) et dans les corollaires de ce texte. Plus précisément, la valeur $\kappa(n)=(n+1)(n+1) !^{n}-n$ donnée dans op. cit. doit être remplacée partout par $\kappa(n)=2 n(n+1) !^{n}-1$ comme indiqué dans l'introduction. 


\section{3. - Le choix des paramètres}

Nous montrons maintenant que si le point $\boldsymbol{\alpha}$ vérifie les hypothèses du Théorème 1.5 , on peut choisir convenablement des entiers $L$ et $T$ de telle sorte que la fonction construite à l'aide de la Proposition 2.1 vérifie les hypothèses du Lemme 2.4 ci-dessus pour «beaucoup» de nombres premiers. Soit $\boldsymbol{\alpha}$ un point de $\mathbb{G}_{m}^{n}$; on notera dans toute la suite de ce Paragraphe 2 :

$$
\omega:=\omega_{K}(\boldsymbol{\alpha}), \quad \omega_{*}:=3[K: \mathbb{Q}] \omega .
$$

On choisit les paramètres $L$ et $T$ :

$$
L:=\left[C_{0}^{\frac{1}{2}} \omega\left(\frac{\log \omega_{*}}{\log \log \omega_{*}}\right)^{2 n}\right], \quad T:=\left[C_{0}^{\frac{1}{4}}\left(\frac{\log \omega_{*}}{\log \log \omega_{*}}\right)^{n}\right] .
$$

Soit de plus $\rho$ un nombre réel tel que $0 \leq \rho<(n+1) !^{n-1}$. On introduit maintenant :

$$
N_{j}:=\left(C_{0} \log \omega_{*}\right)^{2(\rho+1) n j \cdot j !}, \quad j=1, \ldots, n .
$$

Définissons pour finir $n$ ensembles d'entiers, en posant :

$\mathcal{P}_{j}:=\{1\} \cup\left\{p\right.$ premier, $\left.\log \omega_{*} \leq p \leq N_{j}\right\} \backslash\left\{p\right.$ premier, $\left.p \mid \mathfrak{f}_{K}\right\}, \quad j=1, \ldots, n$.

Ci-dessus, $C_{0}$ désigne un nombre réel $>0$, ne dépendant que de $n$, «suffisamment grand» (en d'autres termes, les inégalités que nous serons amenés à écrire seront vraies asymptotiquement en $C_{0}$ ).

On notera aussi $c_{1}, c_{2}, \ldots$, des nombres réels $>0$ (effectivement calculables) ne dépendant que de $n$.

Notons que l'on a $\sum_{k=1}^{j} k \cdot k !=(j+1) !-1$; on en déduit la relation suivante que nous utiliserons plusieurs fois par la suite :

$$
N_{1} \ldots N_{j}=\left(C_{0} \log \omega_{*}\right)^{2(\rho+1) n((j+1) !-1)},
$$

$(j=1, \ldots, n)$. Notons que l'on a aussi :

$$
\log \omega \leq \log (L+1) \leq c_{1}\left(\log C_{0}\right) \log \omega_{*},
$$

et :

$$
\log \left(N_{i}\right) \leq c_{2} \log \left(C_{0}\right) \log \log \omega_{*} .
$$

La proposition suivante, résume l'étape de transcendance :

Proposition 2.5. Supposons que :

$$
h(\boldsymbol{\alpha})<\frac{1}{\omega\left(C_{0} \log \omega_{*}\right)^{2(\rho+1) n((n+1) !-1)+2 n-1}} ;
$$

il existe alors un polynôme non nul $F \in \mathcal{O}_{K}\left[x_{1}, \ldots, x_{n}\right]$ de degré $\leq L$, tel que $F^{\phi_{1} \ldots \phi_{p_{n}}}$ soit nul en $\boldsymbol{\alpha}^{p_{1} \ldots p_{n}}$ pour tout $\left(p_{1}, \ldots, p_{n}\right) \in \mathcal{P}_{1} \times \ldots \times \mathcal{P}_{n}$. 
DÉMONSTRATiOn. Notons pour simplifier :

$$
\Delta=\frac{\log \omega_{*}}{\log \log \omega_{*}} .
$$

On a alors :

$$
\frac{2 \omega T}{L} \leq \frac{2 \omega \cdot C_{0}^{\frac{1}{4}} \Delta^{n}}{\left(C_{0}^{\frac{1}{2}} / 2\right) \omega \Delta^{2 n}}=\frac{4}{C_{0}^{\frac{1}{4}} \Delta^{n}}<1,
$$

et, grâce à l'hypothèse (6),

$$
\frac{L}{T \log (L+1)} h(\boldsymbol{\alpha})<\frac{C_{0}^{\frac{1}{2}} \omega \Delta^{2 n}}{\left(C_{0}^{\frac{1}{4}} / 2\right) \Delta^{n} \cdot \log \omega} h(\boldsymbol{\alpha}) \leq \frac{2 C_{0}^{\frac{1}{4}} \omega \Delta^{n}}{\log \omega} h(\boldsymbol{\alpha})<1,
$$

ce qui montre que les hypothèses de la Proposition 2.1 sont satisfaites.

On déduit donc de cette dernière l'existence de $F \in \mathcal{O}_{K}\left[x_{1}, \ldots, x_{n}\right]$ de degré $\leq L$, s'annulant en $\boldsymbol{\alpha}$ à un ordre $\geq T$ dont la hauteur satisfait l'inégalité (utiliser (4)) :

$$
\begin{aligned}
h(F) & \leq \log [K: \mathbb{Q}]+\frac{4^{n+1} \omega T^{2} \log (L+1)}{L} \\
& \leq \log \omega_{*}+\frac{4^{n+1} \omega\left(C_{0}^{\frac{1}{4}} \Delta^{n}\right)^{2} c_{1}\left(\log C_{0}\right) \log \omega_{*}}{\left(C_{0}^{\frac{1}{2}} / 2\right) \omega \Delta^{2 n}} \\
& \leq c_{3}\left(\log C_{0}\right) \log \omega_{*} .
\end{aligned}
$$

Soit $\left(q_{1}, \ldots, q_{n}\right) \in \mathcal{P}_{1} \times \ldots \times \mathcal{P}_{n}$ et supposons $q_{j_{1}}, \ldots, q_{j_{r}}$ premiers et $q_{j}=1$ pour $j \notin\left\{j_{1}, \ldots, j_{r}\right\}$. Notons $p_{i}=q_{j_{i}}(i=1, \ldots, r)$. On a (en utilisant à nouveau (4)) :

$$
2^{r-1} \prod_{j=1}^{r-1}\left(1+\frac{(n+1) \log (L+1)}{\log p_{j}}\right) \leq c_{4}\left(\log C_{0}\right)^{n-1} \Delta^{n-1}
$$

et donc :

$$
\left(T_{r-1} \min _{1 \leq j \leq r} \log p_{j}\right)^{-1} \leq \frac{c_{4}\left(\log C_{0}\right)^{n-1} \Delta^{n-1}}{\left(C_{0}^{\frac{1}{4}} / 2\right) \Delta^{n} \cdot \log \log \omega_{*}}=\frac{2 c_{4}\left(\log C_{0}\right)^{n-1}}{C_{0}^{\frac{1}{4}} \Delta \cdot \log \log \omega_{*}},
$$

où $T_{r-1}$ est la quantité définie dans l'énoncé du Lemme 2.4. Un calcul facile permet maintenant de vérifier que les hypothèses de ce lemme sont satisfaites ; en effet, on a :

$$
\frac{4 h(F)}{T_{r-1} \min _{1 \leq j \leq r} \log p_{j}} \leq \frac{8 c_{3} c_{4}\left(\log C_{0}\right)^{n} \log \omega_{*}}{C_{0}^{\frac{1}{4}} \Delta \cdot \log \log \omega_{*}}=\frac{8 c_{3} c_{4}\left(\log C_{0}\right)^{n}}{C_{0}^{\frac{1}{4}}}<1 .
$$


et, en tenant compte de la relation (3) :

$$
\begin{aligned}
\frac{4 L p_{j_{1}} \ldots p_{j_{r}}}{T_{r-1} \min _{1 \leq j \leq r} \log p_{j}} h(\boldsymbol{\alpha})< & \frac{4 C_{0}^{\frac{1}{2}} \omega \Delta^{2 n} \cdot N_{j_{1}} \ldots N_{j_{r}} \cdot 2 c_{4}\left(\log C_{0}\right)^{n-1}}{C_{0}^{\frac{1}{4}} \Delta \cdot \log \log \omega_{*}} h(\boldsymbol{\alpha}) \\
\leq & \frac{8 c_{4} C_{0}^{\frac{1}{4}}\left(\log C_{0}\right)^{n-1} \omega \Delta^{2 n-1}}{\log \log \omega_{*}} N_{1} \ldots N_{n} h(\boldsymbol{\alpha}) \\
= & \frac{8 c_{4} C_{0}^{\frac{1}{4}}\left(\log C_{0}\right)^{n-1} \omega \Delta^{2 n-1}}{\log \log \omega_{*}} \\
& \times\left(C_{0} \log \omega_{*}\right)^{2(\rho+1) n((n+1) !-1)} h(\boldsymbol{\alpha}) \\
\leq & c_{5} C_{0}^{\frac{1}{4}}\left(\log C_{0}\right)^{n-1} \omega\left(\log \omega_{*}\right)^{2 n-1} \\
& \times\left(C_{0} \log \omega_{*}\right)^{2(\rho+1) n((n+1) !-1)} \\
& \times \omega^{-1}\left(C_{0} \log \omega_{*}\right)^{-2(\rho+1) n((n+1) !-1)-(2 n-1)} \\
= & c_{5} C_{0}^{\frac{1}{4}}\left(\log C_{0}\right)^{n-1} C_{0}^{-(2 n-1)}<1 .
\end{aligned}
$$

Nous pouvons donc bien appliquer le Lemme 2.4 qui nous assure que $F^{\phi_{p_{1}} \ldots \phi_{p_{r}}}$ est nul en $\boldsymbol{\alpha}^{p_{1} \ldots p_{r}}$ à un ordre $\geq T_{r}$, où :

$$
T_{r}=2^{-r} \prod_{j=1}^{r}\left(1+\frac{(n+1) \log (L+1)}{\log p_{j}}\right)^{-1} \times T .
$$

Comme auparavant, on a :

$$
2^{r} \prod_{j=1}^{r}\left(1+\frac{(n+1) \log (L+1)}{\log p_{j}}\right) \leq c_{6}\left(\log C_{0}\right)^{n} \Delta^{n},
$$

d'où :

$$
T_{r} \geq \frac{\left(C_{0}^{\frac{1}{4}} / 2\right) \Delta^{n}}{\left(\log C_{0}\right)^{n} \Delta^{n}} \geq 1 .
$$

Nous avons bien montré que $F^{\phi_{q_{1}} \ldots \phi_{q_{n}}}$ est nul en $\boldsymbol{\alpha}^{q_{1} \ldots q_{n}}$ pour tout $\left(q_{1}, \ldots, q_{n}\right) \in$ $\mathcal{P}_{1} \times \ldots \times \mathcal{P}_{n}$, ce qui établie la Proposition 2.5.

\section{4. - Lemme de zéros}

Nous allons maintenant utiliser une variante sur $K$ du Théorème 3.2 de [Am-Da] (lemme de zéros avec isogénies); on pourra se reporter à [Da-Hi], théorème 4.10 pour un énoncé plus général, valable pour un groupe algébrique commutatif quelconque sur un corps de nombres (et plus généralement sur un corps de caractéristique zéro). 
THÉORÈME 2.6. Soit $F \in K\left[x_{1}, \ldots, x_{n}\right]$ un polynôme non identiquement nul de degré $\leq L$, tel que $F^{\phi_{p_{1}} \ldots \phi_{p_{n}}}\left(\boldsymbol{\alpha}^{p_{j} \ldots p_{n}}\right)=0$ pour $\left(p_{j}, \ldots, p_{n}\right) \in \mathcal{P}_{j} \times \ldots \times \mathcal{P}_{n}$. Il existe alors un entier $r$ compris entre 1 et $n$, une sous-variété algébrique $V$ propre et $K$-irréductible de $\mathbb{G}_{m}^{n}$ et $\left(p_{r+1}, \ldots, p_{n}\right) \in \mathcal{P}_{r+1} \times \ldots \times \mathcal{P}_{n}$ tels que

$$
\boldsymbol{\alpha}^{p_{r+1} \ldots p_{n}} \in \phi_{p_{r+1}} \ldots \phi_{p_{n}} V,
$$

et tels que de plus :

$$
\operatorname{deg}\left(\bigcup_{p \in \mathcal{P}_{r}} \phi_{p}^{-1}[p] V\right) \leq\left(N_{1} \ldots N_{r-1} L\right)^{\operatorname{codim}(V)} .
$$

Enfin, on peut supposer $\operatorname{codim}(V) \leq r$.

Démonstration. On reprend les notations du Paragraphe 3.2 de [Am-Da] et la preuve du Théorème 3.2 de op. cit. On note $\mathfrak{I}_{1}=(F)$ et, pour $2 \leq r \leq n+1$,

$$
\mathfrak{I}_{r}=\left(G^{\phi_{p}}\left(\mathbf{x}^{p}\right) ; \quad G \in \mathfrak{I}_{r-1}, \quad p \in \mathcal{P}_{r-1}\right) .
$$

Notons $Y_{r}$ la réunion des composantes $V$ de l'ensemble algébrique $\mathcal{Z}\left(\mathfrak{I}_{r}\right)$ tels que :

$$
\exists\left(p_{r}, \ldots, p_{n}\right) \in \mathcal{P}_{r} \times \ldots \times \mathcal{P}_{n} \text { tel que } \boldsymbol{\alpha}^{p_{r} \ldots p_{n}} \in \phi_{p_{r}} \ldots \phi_{p_{n}} V .
$$

On vérifie alors, comme dans loc. cit., que $Y_{r} \neq \emptyset$ et que $[p] Y_{r+1} \subset \phi_{p} Y_{r}$ pour tout $p \in \mathcal{P}_{r}$. On en déduit, toujours comme dans loc. cit., l'existence d'un entier $r$ tel que $\operatorname{codim}\left(Y_{r}\right) \leq r \leq n$ pour lequel $Y_{r}$ et $Y_{r+1}$ ont la même dimension notée $d$. Si $r$ est choisi minimal parmi les entiers $l$ tels que $\operatorname{dim}\left(Y_{l}\right)=\operatorname{dim}\left(Y_{l+1}\right)$, on a bien $n-d \leq r$. Soit donc $V$ une composante irréductible de dimension $d$ de $Y_{r+1}$; les inclusions $[p] Y_{r+1} \subset \phi_{p} Y_{r}\left(p \in \mathcal{P}_{r}\right)$ et la Proposition $3.3 \mathrm{de}[\mathrm{Ph}]$ montrent alors l'inégalité (7).

Afin de pouvoir tirer parti du lemme de zéros, nous utilisons la version suivante du Lemme 5.2 de [Am-Da] :

Lemme 2.7. Soit $W$ une sous variété définie sur $K$ et $K$-irréductible quelconque de $\mathbb{G}_{m}^{n}$ telle que :

$$
\log \operatorname{deg}(W) \leq\left(C_{0} \log \omega_{*}\right)^{2(\rho+1) n-1} .
$$

Pour tout entier $j, 1 \leq j \leq n$, posons $\mathcal{P}_{j}(W):=\mathcal{P}_{j} \backslash E(W)$. On a alors :

$$
\left|\mathcal{P}_{j}(W)\right| \geq c_{7} \frac{\left(C_{0} \log \omega_{*}\right)^{2(\rho+1) n j . j !}}{\left(\log C_{0}\right) \log \log \omega_{*}} .
$$

DÉMONSTRATION. L'argument de loc. cit. se recopie mutatis mutandis une fois noté que le nombre de premiers divisant $\mathfrak{f}_{K}$ est au plus $\log \left(\mathfrak{f}_{K}\right) \leq \log \left(\omega_{\star}\right)$. 
Nous montrons maintenant que si le Théorème 1.5 est faux, on peut trouver de petits multiples de $\boldsymbol{\alpha}$ dont l'indice d'obstruction est beaucoup plus faible que celui de $\alpha$ :

Proposition 2.8. Supposons que l'inégalité (hypothèse (6)) :

$$
h(\boldsymbol{\alpha})<\frac{1}{\omega\left(C_{0} \log \omega_{*}\right)^{2(\rho+1) n((n+1) !-1)+2 n-1},}
$$

soit satisfaite. Supposons de plus qu'il n'existe pas de sous-variétés de torsion B de $\mathbb{G}_{m}^{n}$ définie sur $K$ et passant par un conjugué de $\boldsymbol{\alpha}$ telles que

$$
(\operatorname{deg} B)^{1 / \operatorname{codim}(B)} \leq \omega\left(C_{0} \log \omega_{*}\right)^{4 n((\rho+1)(n+1) !-1)} .
$$

Soit enfin $W$ une sous-variété quelconque de $\mathbb{G}_{m}^{n}$, définie sur $K$ et $K$-irréductible dont le degré satisfait l'inégalité (8) :

$$
\log ([K: \mathbb{Q}] \operatorname{deg}(W)) \leq\left(C_{0} \log \omega_{*}\right)^{2(\rho+1) n-1} .
$$

Il existe alors un entier $l \notin E(W)$ tel que,

$$
l \leq\left(C_{0} \log \omega_{*}\right)^{2(\rho+1) n((n+1) !-1)},
$$

et tel que :

$$
\omega_{K}\left(\boldsymbol{\alpha}^{l}\right)<\frac{\omega}{C_{0}\left(C_{0} \log \omega_{*}\right)^{2 \rho n}} .
$$

DÉmonstration. On suit la preuve de la Proposition 5.3 de [Am-Da]. Soit $F$ le polynôme de degré $\leq L$ fourni par la Proposition 2.5. Ce polynôme satisfait en particulier les hypothèses du lemme de zéros (Théorème 2.6), appliqué aux nouveaux ensembles d'entiers $\mathcal{P}_{j}(W)$ fixés au Lemme 2.7. Le lemme de zéros nous fournit donc un indice $r$ et une sous-variété $V$ de $\mathbb{G}_{m}^{n}$, définie sur $K$ et $K$-irréductible, passant par un conjugué d'une certaine puissance $\boldsymbol{\alpha}^{l}$, avec

$$
l \notin E(W)
$$

(confer Proposition 1.7, point (ii)) et :

$$
l \leq N_{r+1} \ldots N_{n} \leq N_{1} \ldots N_{n}=\left(C_{0} \log \omega_{*}\right)^{2 n(\rho+1)((n+1) !-1)},
$$

pour laquelle la conclusion du lemme de zéros (inégalité (7)) est satisfaite. L'entier $l$ étant maintenant fixé, il reste à estimer $\omega_{K}\left(\boldsymbol{\alpha}^{l}\right)$. Pour ce faire, notons que cette inégalité donne en particulier (car $1 \in \mathcal{P}_{r}(W)$ ) :

$$
\begin{aligned}
\operatorname{deg}(V)^{1 / \operatorname{codim}(V)} & \leq L N_{1} \ldots N_{r-1} \\
& \leq c_{8} C_{0}^{\frac{1}{2}} \omega\left(\log \omega_{*}\right)^{2 n}\left(C_{0} \log \omega_{*}\right)^{2 n(\rho+1)(r !-1)} \\
& \leq \omega\left(C_{0} \log \omega_{*}\right)^{2 n((\rho+1)(n !-1)+1)} \\
& \leq \omega\left(C_{0} \log \omega_{*}\right)^{2 n((\rho+1)(n+1) !-1)}
\end{aligned}
$$


La variété $B=[l]^{-1} V$ passe par un conjugué de $\alpha$ et son degré satisfait l'inégalité :

$$
\operatorname{deg}(B)^{1 / \operatorname{codim}(B)} \leq l \operatorname{deg}(V)^{1 / \operatorname{codim}(V)} \leq \omega\left(C_{0} \log \omega_{*}\right)^{4 n((\rho+1)(n+1) !-1)} .
$$

Par hypothèse, la variété $B$ (et donc $V$ ) n'est pas de torsion. Par ailleurs, l'inégalité (10) montre en particulier que :

$$
\log ([K: \mathbb{Q}] \operatorname{deg}(V)) \leq c_{9}\left(\log C_{0}\right) \log \omega_{*} .
$$

Soit $\mathcal{Q}:=\mathcal{P}_{r}(W) \backslash E(V)$; la Proposition 1.7, point (i) et l'inégalité ci-dessus assurent que $|E(V)|$ est négligeable devant la minoration de $\left|\mathcal{P}_{j}(W)\right|$ fournie par le Lemme 2.7 ; on a donc :

$$
|\mathcal{Q}| \geq c_{10} \frac{\left(C_{0} \log \omega_{*}\right)^{2(\rho+1) n r . r !}}{\log \left(C_{0}\right) \log \log \omega_{*}} .
$$

Soit $W$ une composante $\overline{\mathbb{Q}}$-irréductible de $V$. Comme cette dernière n'est pas de torsion, le Lemme 2.3, point (i), de [Am-Da] montre que les variétés $\phi_{p}^{-1}[p] V$ et $\phi_{q}^{-1}[q] V\left(p, q \in \mathcal{P}_{r}(W), p \neq q\right)$ n'ont pas des composantes communes. Par ailleurs, $\mathcal{Q} \cap E(V)=\emptyset$ et donc (confer définition 1.6) $\operatorname{deg}\left(\phi_{p}^{-1}[p] V\right) \geq \operatorname{deg}(V)$, pour $p \in \mathcal{Q}$. En tenant compte encore de l'inégalité (7) du lemme de zéros, on obtient donc :

$$
|\mathcal{Q}| \operatorname{deg}(V) \leq \operatorname{deg}\left(\bigcup_{p \in \mathcal{P}_{r}(W)} \phi_{p}^{-1}[p] V\right) \leq\left(L N_{1} \ldots N_{r-1}\right)^{\operatorname{codim}(V)} .
$$

En tenant compte de l'inégalité $\operatorname{codim}(V) \leq r$ (confer Théorème 2.6), on obtient la majoration suivante pour le degré de $V$ :

$$
\operatorname{deg}(V)^{1 / \operatorname{codim}(V)} \leq L N_{1} \ldots N_{r-1} \cdot|\mathcal{Q}|^{-1 / r} .
$$

On utilise maintenant la relation (3) pour estimer $N_{1} \ldots N_{r-1}$, ainsi que la majoration de $|\mathcal{Q}|$ obtenue ci-dessus et l'on en déduit :

$$
\begin{aligned}
\operatorname{deg}(V)^{1 / \operatorname{codim}(V)} & \leq L N_{1} \ldots N_{r-1} \cdot|\mathcal{Q}|^{-1 / r} \\
& \leq \frac{c_{11} C_{0}^{\frac{1}{2}} \omega\left(\log \omega_{*}\right)^{2 n}\left(C_{0} \log \omega_{*}\right)^{2(\rho+1) n(r !-1)}\left(\log \left(C_{0}\right) \log \log \omega_{*}\right)^{1 / r}}{(\log \log 3 \omega)^{2 n}\left(C_{0} \log \omega_{*}\right)^{2(\rho+1) n r !}} \\
& =\frac{c_{11}\left(\log \left(C_{0}\right) \log \log \omega_{*}\right)^{1 / r} \omega}{C_{0}^{n+\frac{1}{2}}(\log \log 3 \omega)^{2 n}\left(C_{0} \log \omega_{*}\right)^{2 \rho n}} \leq \frac{\omega}{n C_{0}\left(C_{0} \log \omega_{*}\right)^{2 \rho n}} .
\end{aligned}
$$

Pour finir, comme $\omega_{K}(V) \leq n \operatorname{deg}(V)^{1 / \operatorname{codim}(V)}$ (confer [Ch], Corollaire 2, Chapitre 1 , page 8 et exemple 1, page 9 ), on obtient :

$$
\omega_{K}\left(\boldsymbol{\alpha}^{l}\right) \leq \omega_{K}(V) \leq n(\operatorname{deg}(V))^{1 / \operatorname{codim}(V)} \leq \frac{\omega}{C_{0}\left(C_{0} \log \omega_{*}\right)^{2 \rho n}} .
$$

La Proposition 2.8 est donc entièrement établie. 


\section{3. - La descente finale}

Si dans la Proposition 2.8 qui précède, on pouvait assurer $l=1$, le Théorème 1.5 s'en déduirait immédiatement. Ce n'est malheureusement pas le cas et nous avons recours à essentiellement le même argument de «descente» que dans [Am-Da]. Ce dernier permet, à l'aide d'estimations fines du stabilisateur de certaines variétés de montrer que «l'on ne peut appliquer plus de $n$ fois» de suite la Proposition 2.8.

Soit $K$ une extension cyclotomique de $\mathbb{Q}$ et $\boldsymbol{\alpha} \in \mathbb{G}_{m}^{n}$. Notons comme auparavant

$$
\omega=\omega_{K}(\boldsymbol{\alpha}), \quad \omega_{*}=3[K: \mathbb{Q}] \omega_{K}(\boldsymbol{\alpha}) .
$$

La dernière étape de la preuve est très similaire au Paragraphe 5 de [Am-Da]. On commence par fixer $2 n$ paramètres $(i=1, \ldots, n)$ :

$$
\left\{\begin{array}{l}
\varepsilon_{i}=\frac{1}{C_{0}\left(C_{0} \log \left(\omega_{*}\right)\right)^{2 n\left((n+1) !^{n-i}-1\right)}}, \\
L_{i}=\left(C_{0}\left(\log C_{0}\right)^{2} \log \left(\omega_{*}\right)\right)^{2 n(n+1) !^{n-i}((n+1) !-1)} .
\end{array}\right.
$$

Remarquons que l'on dispose des relations suivantes (confer [Am-Da], Lemme 5.4), qui nous seront utiles dans la suite :

$$
\begin{aligned}
L_{1} \ldots L_{i} & =\left(C_{0}\left(\log C_{0}\right)^{2} \log \left(\omega_{*}\right)\right)^{2 n\left((n+1) !^{n}-(n+1) !^{n-i}\right)} ; \\
L_{i+1} \ldots L_{n} & =\left(C_{0}\left(\log C_{0}\right)^{2} \log \left(\omega_{*}\right)\right)^{2 n\left((n+1) !^{n-i}-1\right)} .
\end{aligned}
$$

Si comme indiqué on pouvait «appliquer $n$ fois de suite» la Proposition 2.8, on se retrouverait avec des suites de variétés vérifiant des propriétés particulières, motivant la définition suivante calquée sur la définition 5.1 de [Am-Da].

DÉFInItion 3.1. On note $\mathcal{W}$ l'ensemble des triplets $(k, \mathbf{l}, \mathbf{V})$, où $k \in[0, n]$ est un entier, $\mathbf{I}=\left(l_{1}, \ldots, l_{k}\right)$ est un $k$-uplet d'entiers avec $0 \leq l_{i} \leq L_{i}$, et $\mathbf{V}=\left(V_{0}, \ldots, V_{k}\right)$ est un $(k+1)$-uplet de sous-variétés propres de $\mathbb{G}_{m}^{n}$, définies sur $K$ et $K$-irréductibles, telles que $\alpha \in V_{0}$ et telles que :

(i) $l_{i} \notin E\left(V_{i-1}\right)$ et $\left[l_{i}\right]^{-1} V_{i}$ contient $V_{i-1}$ pour $i=1, \ldots, k$.

(ii) Pour $i=0, \ldots, k$, l'on a :

$$
\operatorname{deg}\left(V_{i}\right) \leq\left(L_{i+1} \ldots L_{n} \omega_{K}\left(\boldsymbol{\alpha}^{l_{1} \ldots l_{i}}\right)\right)^{\operatorname{codim}\left(V_{i}\right)} .
$$

(iii) Pour $i=1, \ldots, k$, l'on a :

$$
\omega_{K}\left(\boldsymbol{\alpha}^{l_{1} \ldots l_{i}}\right) \leq \varepsilon_{i} \omega_{K}\left(\boldsymbol{\alpha}^{l_{1} \ldots l_{i-1}}\right) .
$$

Enfin, on note $\mathcal{W}_{0}$ le sous ensemble suivant de $\mathcal{W}$ :

$$
\mathcal{W}_{0}:=\left\{(k, \mathbf{l}, \mathbf{V}) \in \mathcal{W}, \operatorname{dim}\left(V_{0}\right)<\operatorname{dim}\left(V_{1}\right)<\ldots<\operatorname{dim}\left(V_{k}\right)\right\}
$$


Comme pour tout $i \leq n$, on a $\varepsilon_{i}<1$, on a en particulier pour tout $(k, \mathbf{l}, \mathbf{V}) \in \mathcal{W}:$

$$
\omega_{K}\left(\boldsymbol{\alpha}^{l_{1} \ldots l_{i}}\right) \leq \omega_{K}\left(\boldsymbol{\alpha}^{l_{1} \ldots l_{i-1}}\right), \quad i=1, \ldots, k .
$$

Introduisons enfin, comme dans loc. cit., une relation d'ordre total sur les suites finies de longueur $\leq n+1$ d'entiers positifs ou nuls et strictement inférieurs à $n$. Soient $(v)=\left(v_{i}\right)_{0 \leq i \leq k}$, et $\left(v^{\prime}\right)=\left(v_{j}^{\prime}\right)_{0 \leq j \leq k^{\prime}}$ deux telles suites. On dira que $(v) \preccurlyeq\left(v^{\prime}\right)$ si

$$
\left(v_{i}\right)_{0 \leq i \leq \min \left\{k, k^{\prime}\right\}}<\left(v_{i}^{\prime}\right)_{0 \leq i \leq \min \left\{k, k^{\prime}\right\}}
$$

pour l'ordre lexicographique, ou, si $\left(v_{i}\right)_{0 \leq i \leq \min \left\{k, k^{\prime}\right\}}=\left(v_{i}^{\prime}\right)_{0 \leq i \leq \min \left\{k, k^{\prime}\right\}}$ et si la longueur $k$ de la suite $(v)$ est $\geq$ à la longueur $k^{\prime}$ de la suite $\left(v^{\prime}\right)$.

Avec ces notations et conventions, on dispose de la proposition suivante :

Proposition 3.2. Soit $\boldsymbol{\alpha}$ un point de $\mathbb{G}_{m}^{n}$; supposons qu'il n'existe pas de sous-variétés propres $Z$ de $\mathbb{G}_{m}^{n}$ passant par $\alpha$, qui sont de torsion, définies sur $K$ et $K$-irréductibles, telles que:

$$
(\operatorname{deg} B)^{1 / \operatorname{codim}(B)} \leq \omega\left(C_{0}\left(\log C_{0}\right)^{2} \log \omega_{*}\right)^{2 n\left((n+1) !^{n}+(n+1) ! !^{n-1}-2\right)} .
$$

Supposons de plus que :

$$
h(\boldsymbol{\alpha})<\frac{1}{\omega\left(C_{0}\left(\log C_{0}\right)^{2} \log \left(\omega_{*}\right)\right)^{2 n(n+1) ! n}-1} .
$$

Alors, $\mathcal{W}_{0} \neq \mathcal{W}$.

DÉmonstration. Remarquons tout d'abord que $\mathcal{W}_{0} \neq \emptyset$. En effet, soit $V_{0}$ une hypersurface de $\mathbb{G}_{m}^{n}$, définie sur $K$ et $K$-irréductible, passant par $\boldsymbol{\alpha}$, telle que $\operatorname{deg}\left(V_{0}\right)=\omega$ : le triplet $\left(0, \emptyset,\left(V_{0}\right)\right)$ appartient alors à $\mathcal{W}_{0}$.

Comme l'ensemble des suites finies d'entiers compris entre 0 et $n-1$, de longueur au plus $n$, strictement croissantes (au sens usuel) est fini (en fait de cardinal $\left.\leq 2^{n}-1\right)$, il existe un élément $(k, \mathbf{l}, \mathbf{W})$ de $\mathcal{W}_{0}$ tel que la suite $\left(\operatorname{dim}\left(W_{i}\right)\right)_{0 \leq i \leq k}$ soit minimale pour $\preccurlyeq$.

Fixons un tel triplet, et remarquons en particulier que $k \leq n-1$ (sinon, par le principe des tiroirs, il existerait un indice $i, 1 \leq i \leq k$ pour lequel $\left.\operatorname{dim}\left(W_{i-1}\right)=\operatorname{dim}\left(W_{i}\right)\right)$.

On se propose d'utiliser la Proposition 2.8 avec :

$$
\rho=(n+1) !^{n-1-k}-1, \quad W=W_{k},
$$

et $\boldsymbol{\alpha}$ remplacé par $\boldsymbol{\alpha}^{l_{1} \ldots l_{k}}$. On a $h\left(\boldsymbol{\alpha}^{l_{1} \ldots l_{k}}\right)=l_{1} \ldots l_{k} h(\boldsymbol{\alpha})$ et $\omega_{K}\left(\boldsymbol{\alpha}^{l_{1} \ldots l_{k}}\right) \leq \omega_{K}(\boldsymbol{\alpha})$ (par la relation (13)). Donc, en utilisant la formule (11) pour estimer $\prod_{i} L_{i}$ et l'hypothèse sur $h(\boldsymbol{\alpha})$, on a :

$$
\begin{aligned}
h\left(\boldsymbol{\alpha}^{l_{1} \ldots l_{k}}\right) \omega_{K}\left(\boldsymbol{\alpha}^{l_{1} \ldots l_{k}}\right) & \leq \omega \cdot L_{1} \ldots L_{k} \cdot h(\boldsymbol{\alpha}) \\
& \leq \frac{\left.\omega\left(C_{0}\left(\log C_{0}\right)^{2} \log \left(\omega_{*}\right)\right)^{2 n\left((n+1) ! !^{n}-(n+1) ! n-k\right.}\right)}{\omega\left(C_{0} \log \left(C_{0}\right)^{2} \log \left(\omega_{*}\right)\right)^{2 n(n+1) ! !^{n}-1}} \\
& =\left(C_{0}\left(\log C_{0}\right)^{2} \log \left(\omega_{*}\right)\right)^{-2 n(n+1) ! !^{n-k}+1} \\
& \leq\left(C_{0} \log \left(\omega_{*}\right)\right)^{-e},
\end{aligned}
$$


avec :

$$
\begin{aligned}
e & =2 n(n+1) !^{n-k}-1 \\
& =2(\rho+1) n((n+1) !-1)+2 n-1+2 \rho n \\
& \geq 2(\rho+1) n((n+1) !-1)+2 n-1 .
\end{aligned}
$$

L'hypothèse sur $h\left(\boldsymbol{\alpha}^{l_{1} \ldots l_{k}}\right)$ de la Proposition 2.8 (relation (6)) est donc satisfaite.

Supposons par l'absurde qu'il existe une variété de torsion $B$ de $\mathbb{G}_{m}^{n}$ définie sur $K$ et passant par $\boldsymbol{\alpha}^{l_{1} \ldots l_{k}}$ qui satisfait la majoration (9). Dans ce cas, posons $B^{\prime}:=\left[l_{1} \ldots l_{k}\right]^{-1} B$; c'est une sous-variété de torsion de $\mathbb{G}_{m}^{n}$, passant par $\boldsymbol{\alpha}$ et dont le degré est :

$$
\operatorname{deg}\left(B^{\prime}\right)=\left(l_{1} \ldots l_{k}\right)^{\operatorname{codim}(B)} \operatorname{deg}(B)
$$

En tenant compte de la formule (11) (pour estimer $\prod_{i} L_{i}$ ) et de l'hypothèse (9) sur le degré de $B$, on a :

$$
\begin{aligned}
\left(\operatorname{deg} B^{\prime}\right)^{1 / \operatorname{codim}\left(B^{\prime}\right) \leq} & L_{1} \ldots L_{k}(\operatorname{deg} B)^{1 / \operatorname{codim}(B)} \\
\leq & \left(C_{0}\left(\log C_{0}\right)^{2} \log \omega_{*}\right)^{2 n\left((n+1) !^{n}-(n+1) !^{n-k}\right)} \\
& \times \omega\left(C_{0} \log \omega_{*}\right)^{4 n\left((n+1) !^{n-1-k}(n+1) !-1\right)} \\
\leq & \omega\left(C_{0}\left(\log C_{0}\right)^{2} \log \omega_{*}\right)^{2 n\left((n+1) !^{n}+(n+1) !^{n-1}-2\right)}
\end{aligned}
$$

ce qui contredit l'hypothèse sur le degré des variétés de torsion contenant éventuellement $\boldsymbol{\alpha}$.

Notons enfin que la variété $W=W_{k}$ vérifie la condition imposée dans la Proposition 2.8. En effet, en utilisant le point (ii) (avec $i=k$ ) de la définition 3.1, l'inégalité (13) (pour majorer $\omega_{K}\left(\boldsymbol{\alpha}^{l_{1} \ldots l_{k}}\right)$ ) et la formule (12) (pour estimer $\prod_{i \geq k+1} L_{i}$ ), on obtient :

$\log \left([K: \mathbb{Q}] \operatorname{deg}\left(W_{k}\right)\right) \leq \log [K: \mathbb{Q}]+n \sum_{i=1}^{n} \log L_{i}+n \log \omega \leq\left(\log C_{0}\right)^{2} \log \left(\omega_{*}\right)$

Les hypothèses de la Proposition 2.8 sont donc satisfaites. On déduit alors de cette dernière qu'il existe un entier $l_{k+1} \notin E\left(W_{k}\right)$, tel que $l_{k+1} \leq L_{k+1}$ et

$$
\omega_{K}\left(\boldsymbol{\alpha}^{l_{1} \ldots l_{k+1}}\right) \leq \varepsilon_{k+1} \omega_{K}\left(\boldsymbol{\alpha}^{l_{1} \ldots l_{k}}\right)
$$

Le triplet $(k, \mathbf{l}, \mathbf{W}) \in \mathcal{W}_{0}$, et satisfait donc en particulier les hypothèses du Lemme 5.9 de [Am-Da] (qui est encore vrai avec $\mathbb{Q}$ remplacé par $K$ et $\delta$ remplacé respectivement par $\omega_{K}$ ). Appliquons ce dernier avec l'entier $l_{k+1}$ donné par la Proposition 2.8 : on en déduit l'existence d'un entier $k^{\prime}, 0 \leq$ $k^{\prime} \leq k+1 \leq n$, et d'une certaine variété propre $Z_{k^{\prime}}$ de $\mathbb{G}_{m}^{n}$, définie sur $K$ et $K$-irréductible, de degré

$$
\leq l_{k^{\prime}+1} \ldots l_{k+1} \omega_{K}\left(\boldsymbol{\alpha}^{l_{1} \ldots l_{k+1}}\right) \operatorname{deg}\left(W_{k^{\prime}}\right)
$$


telle que $\left[l_{k^{\prime}}\right]^{-1} Z_{k^{\prime}} \supset W_{k^{\prime}-1}$ et tel que $\operatorname{codim}\left(Z_{k^{\prime}}\right)=\operatorname{codim}\left(W_{k^{\prime}}\right)+1$ (on a utilisé les conventions : $\operatorname{codim}\left(W_{k+1}\right)=0, \operatorname{deg}\left(W_{k+1}\right)=1, W_{-1}=\{\sigma(\boldsymbol{\alpha}), \sigma \in$ $\operatorname{Gal}(\overline{\mathbb{Q}} / K)\}$ et $\left.l_{0}=1\right)$. De plus, on a :

$$
\left(\operatorname{dim}\left(W_{0}\right), \ldots, \operatorname{dim}\left(W_{k^{\prime}-1}\right), \operatorname{dim}\left(Z_{k^{\prime}}\right)\right) \prec\left(\operatorname{dim}\left(W_{0}\right), \ldots, \operatorname{dim}\left(W_{k}\right)\right) .
$$

Nous allons maintenant vérifier que :

$$
\left(k^{\prime},\left(l_{1}, \ldots, l_{k^{\prime}}\right),\left(W_{0}, \ldots, W_{k^{\prime}-1}, Z_{k^{\prime}}\right)\right) \in \mathcal{W}
$$

Pour ce faire, il suffit de vérifier les conditions de (i) à (iii) de la définition 3.1 avec $i=k^{\prime}$. La première partie de la condition (i) est assurée par le choix de $l_{k+1}$ si $k^{\prime}=k+1$ et par l'hypothèse $(k, \mathbf{l}, \mathbf{W}) \in \mathcal{W}_{0}$ sinon ; la deuxième partie est assurée par construction de $Z_{k+1}$ (et toujours par hypothèse si $k^{\prime}<k+1$ ). Un argument similaire s'applique pour la condition (iii). Montrons donc (ii). Les relations sur la codimension et sur le degré de $Z_{k^{\prime}}$, la majoration du degré de $W_{k^{\prime}}$ (définition 3.1) et l'inégalité (13), nous assurent que :

$$
\begin{aligned}
\operatorname{deg}\left(Z_{k^{\prime}}\right) & \leq l_{k^{\prime}+1} \ldots l_{k+1} \omega_{K}\left(\boldsymbol{\alpha}^{l_{1} \ldots l_{k+1}}\right) \operatorname{deg}\left(W_{k^{\prime}}\right) \\
& \leq L_{k^{\prime}+1} \ldots L_{n} \omega_{K}\left(\boldsymbol{\alpha}^{l_{1} \ldots l_{k^{\prime}}}\right)\left(L_{k^{\prime}+1} \ldots L_{n} \omega_{K}\left(\boldsymbol{\alpha}^{l_{1} \ldots l_{k^{\prime}}}\right)\right)^{\operatorname{codim}\left(W_{k^{\prime}}\right)} \\
& =\left(L_{k^{\prime}+1} \ldots L_{n} \omega_{K}\left(\boldsymbol{\alpha}^{l_{1} \ldots l_{k^{\prime}}}\right)\right)^{\operatorname{codim}\left(Z_{k^{\prime}}\right)} .
\end{aligned}
$$

Comme la suite des dimensions $\left(\operatorname{dim}\left(W_{0}\right), \ldots, \operatorname{dim}\left(W_{k^{\prime}-1}\right), \operatorname{dim}\left(Z_{k^{\prime}}\right)\right)$ est strictement inférieure à la suite $\left(\operatorname{dim}\left(W_{0}\right), \ldots, \operatorname{dim}\left(W_{k}\right)\right)$ et comme cette dernière est minimale sur les éléments de $\mathcal{W}_{0}$, on en déduit que le triplet :

$$
\left(k^{\prime},\left(l_{1}, \ldots, l_{k^{\prime}}\right),\left(W_{0}, \ldots, W_{k^{\prime}-1}, Z_{k^{\prime}}\right)\right) \in \mathcal{W} \backslash \mathcal{W}_{0},
$$

ce qui établit la Proposition 3.2.

\section{4. - Preuve des théorèmes principaux}

Nous pouvons maintenant établir les Théorèmes 1.4 et 1.5 annoncés dans l'introduction. Le Théorème 1.4 se déduit directement du Théorème 1.5 par «production» de petites relations si la hauteur de $\boldsymbol{\alpha}$ est trop petite ; le Théorème 1.5 s'établit lui directement à partir de la Proposition 3.2 en comparant les degrés des variétés choisies parmi un élément de $\mathcal{W} \backslash \mathcal{W}_{0}$ et en tenant compte de manière essentielle de leur stabilisateur.

DÉmonstration du Théorème 1.5. Soit $K$ une extension cyclotomique de $\mathbb{Q}$, et $\boldsymbol{\alpha} \in \mathbb{G}_{m}^{n}$; pour montrer le Théorème 1.5 , il suffit de supposer les hypothèses de la Proposition 3.2 satisfaites, et d'en déduire une contradiction. Appliquons donc la Proposition 3.2 à $\boldsymbol{\alpha}$; soit $(k, \mathbf{l}, \mathbf{V})$ un élément de $\mathcal{W} \backslash \mathcal{W}_{0}$, 
et soit $i$ un entier $1 \leq i \leq k$ tel que $\operatorname{dim}\left(V_{i-1}\right)=\operatorname{dim}\left(V_{i}\right)$. On sait de plus qu'il existe un élément $l_{i} \notin E\left(V_{i-1}\right)$ tel que :

$$
\left[l_{i}\right]^{-1} V_{i} \supset V_{i-1}
$$

(par la propriété (ii) de la définition 3.1). Notons $W_{1}, \ldots, W_{k}$ les composantes $\overline{\mathbb{Q}}$-irréductibles de $V_{i-1}$; par la définition 1.6 , les variétés $\left[l_{i}\right] W_{a}(a=1, \ldots, k)$ sont deux-à-deux distinctes et sont de degrés $\geq \operatorname{deg}\left(W_{1}\right)$. Donc, $\operatorname{deg}\left(\left[l_{i}\right] V_{i-1}\right) \geq$ $\operatorname{deg}\left(V_{i-1}\right)$. D'autre part, $\left[l_{i}\right] V_{i-1} \subset V_{i}$ et ces variétés ont la même dimension ; on en déduit que :

$$
\operatorname{deg}\left(V_{i-1}\right) \leq \operatorname{deg}\left(V_{i}\right) .
$$

Mais, comme $V_{i-1}$ passe par $\boldsymbol{\alpha}^{l_{1} \ldots l_{i-1}}$, on a :

$$
\omega_{K}\left(\boldsymbol{\alpha}^{l_{1} \ldots l_{i-1}}\right) \leq n \operatorname{deg}\left(V_{i-1}\right)^{1 / \operatorname{codim}\left(V_{i}\right)}
$$

par un résultat de M. Chardin (confer [Ch], Corollaire 2, Chapitre 1, page 8 et exemple 1, page 9). En appliquant les relations (ii) et (iii) de la définition 3.1, on en tire :

$$
\begin{aligned}
\omega_{K}\left(\boldsymbol{\alpha}^{l_{1} \ldots l_{i-1}}\right) & \leq n \operatorname{deg}\left(V_{i-1}\right)^{1 / \operatorname{codim}\left(V_{i-1}\right)} \leq n\left(\operatorname{deg}\left(V_{i}\right)\right)^{1 / \operatorname{codim}\left(V_{i}\right)} \\
& \leq n L_{i+1} \ldots L_{n} \omega_{K}\left(\boldsymbol{\alpha}^{l_{1} \ldots l_{i}}\right) \leq n \varepsilon_{i} L_{i+1} \ldots L_{n} \omega_{K}\left(\boldsymbol{\alpha}^{l_{1} \ldots l_{i-1}}\right) .
\end{aligned}
$$

Enfin, la formule (12) et la définition de $\varepsilon_{i}$ donnent :

$$
\begin{aligned}
\omega_{K}\left(\boldsymbol{\alpha}^{l_{1} \ldots l_{i-1}}\right) & \leq \frac{n\left(C_{0} \log \left(C_{0}\right)^{2} \log \left(\omega_{*}\right)\right)^{2 n\left((n+1) !^{n-i}-1\right)}}{C_{0}\left(C_{0} \log \left(\omega_{*}\right)\right)^{2 n\left((n+1) !^{n-i}-1\right)}} \omega_{K}\left(\boldsymbol{\alpha}^{l_{1} \ldots l_{i-1}}\right) \\
& =\frac{n\left(\log C_{0}\right)^{4 n\left((n+1) !^{n-i}-1\right)}}{C_{0}} \omega_{K}\left(\boldsymbol{\alpha}^{l_{1} \ldots l_{i-1}}\right)<\omega_{K}\left(\boldsymbol{\alpha}^{l_{1} \ldots l_{i-1}}\right) .
\end{aligned}
$$

Cette inégalité est fausse, une contradiction. Les hypothèses de la Proposition 3.2 ne sont donc jamais satisfaites : c'est le Théorème 1.5.

DÉmonstration du Théorème 1.4. On montre le théorème par récurrence sur $n$. Si $n=1$, les Théorèmes 1.5 et 1.4 coïncident (la majoration de l'ordre de la torsion fornie par le Théorème 1.5 est triviale). Supposons donc le théorème vrai pour un certain entier $n-1 \geq 1$ et supposons par l'absurde qu'il soit faux pour $n$. Soit $C_{0}(n)$ un nombre réel assez grand (que l'on pourrait calculer effectivement à partir de la constante $c^{\prime}(n)$ du Théorème 1.5). Il existe alors un entier $\delta \geq 1$, une sous-variété algébrique $V$ de $\mathbb{G}_{m}^{n}$, définie sur un corps cyclotomique $K$ par des polynômes de $K[\mathbf{x}]$ de degré $\leq \delta$ et un point $\boldsymbol{\alpha} \in V$ n'appartenant à aucune sous-variété de torsion contenue dans $V$ et tel que

$$
h(\boldsymbol{\alpha})<C_{0}(n)^{-1} \delta^{-1}(\log (3[K: \mathbb{Q}] \delta))^{-2 n(n+1) !^{n}+1} .
$$


Remarquons que $\omega_{K}(\boldsymbol{\alpha}) \leq \delta$ (puisque $\boldsymbol{\alpha} \in V$ ) : le Théorème 1.5 nous assure donc que $\alpha$ appartient à une certaine sous-variété propre $B$ de $\mathbb{G}_{m}^{n}$, de torsion, définie sur $K$ et $K$-irréductible dont le degré est majoré comme ci-dessus :

$$
(\operatorname{deg} B)^{1 / \operatorname{codim}(B)} \leq c^{\prime}(n) \delta(\log (3[K: \mathbb{Q}] \delta))^{2 n\left((n+1) !^{n}+(n+1) !^{n-1}-2\right)} .
$$

Écrivons

$$
B=\bigcup_{\sigma \in \operatorname{Gal}(K(\zeta) / K)} H \sigma(\zeta)
$$

où $H$ est la composante neutre de $B$ et $\zeta \in \mathbb{G}_{m}^{n}$ est un point de torsion. Quitte à remplacer $\zeta$ par un de ses conjugués, on peut de plus supposer que $\alpha \in H \zeta$. Soit $\Lambda$ le sous-groupe de $\mathbb{Z}^{n}$ des relations de $H$ :

$$
\Lambda=\left\{\boldsymbol{\lambda} \in \mathbb{Z}^{n} \quad \text { tel que } \forall \mathbf{x} \in H, \mathbf{x}^{\boldsymbol{\lambda}}=1\right\} .
$$

On sait que le volume de $\Lambda$ (par rapport à la métrique euclidienne usuelle) est égal au degré de $H$; le théorème de Minkowski nous fournit alors un vecteur non nul $\lambda \in \Lambda$ tel que :

$$
M:=\max _{1 \leq i \leq n}\left\{\left|\lambda_{i}\right|\right\} \leq(\operatorname{deg} H)^{1 / \operatorname{codim}(H)} .
$$

On peut supposer que $\lambda_{1}, \ldots, \lambda_{n}$ sont globalement premiers entre eux (car $H$ est connexe) ; quitte à renuméroter les variables et à changer les signes, on peut également supposer $\lambda_{n}=M$. Le sous-tore $H^{\prime}$ d'équation $\mathbf{x}^{\lambda}=1$ contient donc $H$ et il est paramétré par l'application $\varphi: \mathbb{G}_{m}^{n-1} \rightarrow \mathbb{G}_{m}^{n}$ suivante :

$$
\varphi(\mathbf{x})=\left(x_{1}^{\lambda_{n}}, \ldots, x_{n-1}^{\lambda_{n}}, x_{1}^{-\lambda_{1}} \ldots x_{n-1}^{-\lambda_{n-1}}\right) .
$$

Soit $\boldsymbol{\beta} \in \varphi^{-1}\left(\boldsymbol{\alpha} . \boldsymbol{\zeta}^{-1}\right)$ et notons $V^{\prime}=\varphi^{-1}\left(V \boldsymbol{\zeta}^{-1}\right)$. On a alors :

(a) $V^{\prime} \subsetneq \mathbb{G}_{m}^{n-1}$, car $B \nsubseteq V$ et donc $H^{\prime} \nsubseteq V \zeta^{-1}$. De plus, $V^{\prime}$ est définie sur le corps cyclotomique $K^{\prime}=K(\boldsymbol{\zeta})$ par des polynômes de $K^{\prime}[\mathbf{x}]$ dont le degré satisfait l'inégalité( ${ }^{(3)}$ :

$$
\leq \max \left\{\lambda_{n},\left|\lambda_{1}+\ldots+\lambda_{n-1}\right|\right\} \delta \leq n M \delta
$$

(b) par construction, $\boldsymbol{\beta} \in V^{\prime}$ et $\boldsymbol{\beta}$ n'appartient à aucune sous-variété de torsion incluse dans $V^{\prime}$. En effet, si $\beta \in B^{\prime} \subseteq V^{\prime}$ pour une certaine sous-variété de torsion $B^{\prime}$, alors on aurait $\boldsymbol{\alpha}=\varphi(\boldsymbol{\beta}) \boldsymbol{\zeta} \in \varphi\left(B^{\prime}\right) \boldsymbol{\zeta} \subseteq \varphi\left(V^{\prime}\right) \boldsymbol{\zeta} \subseteq V$, avec $\varphi\left(B^{\prime}\right) \boldsymbol{\zeta}$ de torsion, contre le fait que $\boldsymbol{\alpha}$ n'appartient à aucune sous-variété de torsion contenue dans $V$;

${ }^{(3)}$ On remarquera que, si $Z$ est une hypersurface définie sur $K$, contenante à la fois $V$ et $H^{\prime} \boldsymbol{\zeta}$, alors $\varphi^{-1}\left(Z \boldsymbol{\zeta}^{-1}\right)=\mathbb{G}_{m}^{n-1}$. Donc, on ne peut conclure que $\omega_{K^{\prime}}\left(V^{\prime}\right) \leq n M \omega_{K}(V)$. Pour cette raison nous devons faire intervenir la régularité $\delta$ et non seulement l'indice d'obstruction $\omega_{K}(V)$. 
(c) les hauteurs de $\boldsymbol{\alpha}$ et $\boldsymbol{\beta}$ sont liées par l'inégalité :

$$
h(\boldsymbol{\alpha})=h(\varphi(\boldsymbol{\beta})) \geq h\left(\beta_{1}^{\lambda_{n}}, \ldots, \beta_{n-1}^{\lambda_{n}}\right)=\lambda_{n} h(\boldsymbol{\beta})=M h(\boldsymbol{\beta}) .
$$

Par hypothèse de récurrence, il existe une constante $c(n-1)>0$ telle que

$$
M^{-1} h(\boldsymbol{\alpha}) \geq h(\boldsymbol{\beta}) \geq c(n-1)^{-1}(n M \delta)^{-1}\left(\log \left(3\left[K^{\prime}: \mathbb{Q}\right] . n M \delta\right)\right)^{-2(n-1) n !^{n-1}+1},
$$

d'où, compte tenu de la majoration $M \leq(\operatorname{deg} H)^{1 / \operatorname{codim}(H)} \leq \operatorname{deg}(H)$ et de l'égalité

$$
\operatorname{deg}(B)=\left[K^{\prime}: K\right] \operatorname{deg}(H),
$$

on obtient :

$$
h(\boldsymbol{\alpha}) \geq c(n-1)^{-1} n^{-1} \delta^{-1}(\log (3 n[K: \mathbb{Q}] \operatorname{deg}(B) \delta))^{-2(n-1) n !^{n-1}+1} .
$$

L'inégalité (14) majorant $\operatorname{deg}(B)$, on voit pour finir que l'on peut choisir $C_{0}(n)$ assez grande de telle sorte que :

$n c(n-1)(\log (3 n[K: \mathbb{Q}] \operatorname{deg}(B) \delta))^{2 n(n+1) !^{n}-1} \leq C_{0}(n)(\log (3[K: \mathbb{Q}] \delta))^{2 n(n+1) !^{n}-1}$.

Pour un tel choix de $C_{0}(n)$ on a nécessairement :

$$
\begin{aligned}
h(\boldsymbol{\alpha}) & \geq C_{0}(n)^{-1} \delta^{-1}(\log 3[K: \mathbb{Q}] \delta)^{-2(n-1) n !^{n-1}+1} \\
& \geq C_{0}(n)^{-1} \delta^{-1}(\log 3[K: \mathbb{Q}] \delta)^{-2 n(n+1) !^{n}+1} .
\end{aligned}
$$

Contradiction. D'où le Théorème 1.4.

\section{REFERENCES}

[Am-Da] F. Amoroso - S. DAvid, Le problème de Lehmer en dimension supérieure, J. reine angew. Math. 513 (1999), 145-179.

[Am-Da2] F. Amoroso - S. DAvid, Minoration de la hauteur normalisée des hypersurfaces, Acta Arith. 92 (2000), 340-366.

[Am-Da3] F. Amoroso - S. DAvid, Densité des points à coordonnées multiplicativement indépendantes, Ramanujan Math. Journal. 5 (2001), 237-246.

[Am-Da4] F. Amoroso - S. DAvid, Minoration de la hauteur normalisée dans un tore, Journal de l'Institut de Mathématiques de Jussieu 2 (2003), 335-381.

[Am-Dv] F. Amoroso - R. Dvornicich, A lower bound for the height in abelian extensions, J. Number Theory 80 (2000), 260-272.

[Am-Za] F. Amoroso - U. ZAnnier, Minoration de la hauteur normalisée dans un tore, Ann. Scuola Norm. Sup. Pisa Cl. Sci. (4) 29 (2000), 711-727.

[Bo-Va] E. Bombieri - J. VAALER, Siegel's lemma, Invent. Math. 73 (1983), 11-32. 
[Bo-Za] E. Bombieri - U. ZANnier, Algebraic points on subvarieties of $\mathbb{G}_{m}^{n}$, Int. Math. Res. Not. 7 (1995), 333-347.

[Ch] M. ChARDIN, Une majoration de la fonction de Hilbert et ses conséquences pourl'interpolation algébrique, Bulletin de la Société Mathématique de France 117 (1988), 305-318. Voir aussi Contributions à l'algèbre commutative effective et à la théorie de l'élimination, Thèse de doctorat, Université de Paris VI, 1990.

[Da-Hi] S. DAVID - M. HINDRY, Minoration de la hauteur de Néron-Tate sur les variétés abéliennes de type C.M., J. reine angew. Math. 529 (2000), 1-74.

[Da-Ph] S. DAVID - P. PhILIPPON, Minorations des hauteurs normalisées des sous-variétés des tores, Ann. Scuola Norm. Sup. Pisa Cl. Sci. (4) 28 (1999), 489-543; Errata ibidem 29 (2000).

[Do] E. Dobrowolski, On a question of Lehmer and the number of irreducible factors of a polynomial, Acta Arith. 34 (1979), 391-401.

[Le] H. LeHMER, Factorisation of some cyclotomic functions, Ann. of Math. 34 (1933), 461-479.

[Ph] P. PhilipPon, Lemmes de zéros dans les groupes algébriques commutatifs, Bull. Soc. Math. France 114 (1986), 355-383; Nouveaux lemmes de zéros dans les groupes algébriques commutatifs, Rocky Mountain J. Math. (3) 26 (1996), 1069-1088.

[Sch] W. Schmidt, Heights of points on subvarieties of $\mathbb{G}_{m}^{n}$, in "Number theory, Séminaire de Théorie des Nombres de Paris" 1993-1994 (S. David editeur) London Math. Soc. Ser. 235 (1996), 157-187, Cambridge University Press.

U. M. R. 6139 (C. N. R. S.)

Laboratoire de Mathématiques Nicolas Oresme

Département de Mathématiques

Université de Caen

Campus II, BP 5186

14032 Caen Cédex, France

amoroso@math.unicaen.fr

U. M. R. 7586 (C. N. R. S.) - U. F. R. 921

Problèmes Diophantiens

Département de Mathématiques

Université Pierre et Marie Curie

4, Place Jussieu

75005 Paris, France

david@math.jussieu.fr 